\title{
Localized Aurora B activity spatially controls non-kinetochore microtubules during spindle assembly
}

\author{
Marvin E. Tanenbaum • René H. Medema
}

Received: 5 July 2011 /Revised: 11 July 2011 / Accepted: 13 July 2011 /Published online: 24 July 2011

(C) The Author(s) 2011. This article is published with open access at Springerlink.com

\begin{abstract}
Efficient spindle assembly involves the generation of spatial cues around chromosomes that locally stabilize microtubule (MT) plus-ends. In addition to the small GTPase Ran, there is evidence that Aurora B kinase might also generate a spatial cue around chromosomes but direct proof for this is still lacking. Here, we find that the Aurora B substrate MCAK localizes to MT plus-ends throughout the mitotic spindle, but its accumulation is strongly reduced on MT plus-ends near chromatin, suggesting that a signal emanating from chromosomes negatively regulates MCAK plus-end binding. Indeed, we show that Aurora $\mathrm{B}$ is the kinase responsible for producing this chromosome-derived signal. These results are the first to visualize spatially restricted Aurora B kinase activity around chromosomes on an endogenous substrate and explain how Aurora B could spatially control the dynamics of non-kinetochore MTs during spindle assembly.
\end{abstract}

\section{Introduction}

Spindle assembly was classically viewed as a process in which MTs are nucleated at centrosomes and are subsequently captured by kinetochores after repeated episodes of MT growth and shrinkage in a process termed "search-andcapture" (Kirschner and Mitchison 1986). However, many

Electronic supplementary material The online version of this article (doi:10.1007/s00412-011-0334-9) contains supplementary material, which is available to authorized users.

M. E. Tanenbaum • R. H. Medema ( $\bowtie)$

Department of Medical Oncology and Cancer Genomics Centre, University Medical Center Utrecht,

Universiteitsweg 100, Str.2.118,

3584 CG Utrecht, The Netherlands

e-mail: r.h.medema@umcutrecht.nl studies have now provided evidence that MT capture is not based on random periods of MT growth and shrinkage but rather that MT growth is strongly biased towards chromosomes (Walczak and Heald 2008). It is thought that chromosomes act as a spatial cue by locally generating a Ran-GTP gradient, which activates a plethora of spindle assembly factors in the vicinity of chromosomes (Clarke and Zhang 2008). In addition to Ran-GTP, several lines of evidence suggest that a second pathway involving the Aurora B kinase might act as a spatial cue to enhance MT stability in the vicinity of chromosomes. First, Aurora B localizes to and is activated at chromosomes (Kelly et al. 2007; Ruchaud et al. 2007; Vader et al. 2006). Second, Aurora B is required for MT formation around chromosomes/kinetochores (Maresca et al. 2009; Sampath et al. 2004; Tulu et al. 2006). Third, binding of the MTdestabilizing protein Op18/Stathmin to tubulin is inhibited in the vicinity of chromosomes through phosphorylation (Niethammer et al. 2004) and Aurora B is thought to be the kinase responsible for this phosphorylation (Gadea and Ruderman 2006), although proof that Aurora B phosphorylates Op18/stathmin in a spatially restricted manner is lacking. Finally, Aurora B does act as a spatial cue at the spindle midzone during anaphase (Fuller et al. 2008) and at centromeres in (pro)metaphase (Liu et al. 2009). Importantly, the signaling gradient at the centromere acts over a very short distance and has only been implicated in regulation of kinetochore MTs (Liu et al. 2009). Therefore, it is still unclear whether Aurora B also acts as a spatial cue around chromosomes for non-kinetochore MTs during spindle assembly, analogous to the Ran-GTP pathway. Indeed, while a gradient of Ran-GTP during spindle assembly has been directly visualized using elegant FRETbased sensors (Kalab et al. 2006; Kalab et al. 2002), as well as on an endogenous substrate (Koffa et al. 2006; Sillje et 
al. 2006), direct observation of spatially restricted Aurora B activity around chromosomes is still lacking.

To assess the spatial activity of Aurora B, we have examined the localization of MCAK, a well-known substrate of Aurora B (Andrews et al. 2004; Lan et al. 2004; Ohi et al. 2004). During mitosis, MCAK localizes to kinetochores/centromeres independently of MTs, as well as to the plus-ends of non-kinetochore MTs (Andrews et al. 2004; Lan et al. 2004; Moore et al. 2005; Ohi et al. 2004; Walczak et al. 1996; Wordeman and Mitchison 1995). At the centromeres/kinetochores, MCAK activity is controlled by Aurora B, either to correct erroneous kinetochore-MT attachments or to limit MT nucleation at chromatin (Andrews et al. 2004; Lan et al. 2004; Ohi et al. 2004; Sampath et al. 2004; Tulu et al. 2006). However, much less is known about the regulation of MT plus-end localizion of MCAK on non-kinetochore MTs. MCAK mutants in which the Aurora B phosphorylation sites have been mutated to phospho-mimicking residues no longer bind to MT plus-ends in interphase (Honnappa et al. 2009; Moore et al. 2005), suggesting that MT plus-end localization of MCAK could be a good readout of local Aurora B activity.

\section{Results}

To visualize MCAK localization on MT plus-ends, we fixed U2OS cells using methanol fixation, which specifically preserves the MT plus-end localized pool of MCAK, in contrast to formaldehyde fixation used in previous studies, which allows visualization of kinetochore/centromere bound MCAK (Andrews et al. 2004; Lan et al. 2004). This approach clearly shows that endogenous MCAK localizes to MT plus-ends (visualized by EB1 staining) during mitosis (Fig. 1a). This staining was specific as it disappeared after MCAK RNAi (Supplemental Fig. S1a). Strikingly, MCAK localization was strongly reduced at MT plus-ends that were in the vicinity of chromosomes as compared to astral MT plus-end or plus-ends near spindle poles (Supplemental Fig. S1b; Fig. 1a-b). Quantification of the amount of MCAK associated with MT plus-ends, revealed a $\sim 4-5$-fold reduction in MCAK levels on chromosome-proximal MTs plus-ends as compared with astral MTs plus-ends (Fig. 1b). The decrease in MCAK binding to chromosome-proximal MT plus-ends was especially clear in cells treated with a kinesin-5 inhibitor S-trityl-L-cysteine (STLC) to induce monopolar spindle formation (Fig. 1c). Line scans through the spindle revealed that MCAK staining at MT plus-ends increased with the distance from the chromosomes (Fig. 1d). Taken together, these results show that endogenous MCAK is a MT plusend tracking protein during mitosis and suggest that a signal emanating from the chromosomes negatively regulates MCAK's association with MT plus-ends.

MCAK can potentially co-localize with kinetochore-MT plus-ends either through binding to EB1 at polymerizing kinetochore MTs or through MT-independent binding to kinetochores (Wordeman and Mitchison 1995). In addition, MCAK binds to the plus-ends of growing non-kinetochore MTs both in interphase and mitosis through direct association with EB1 and TIP150 (Honnappa et al. 2009; Jiang et al. 2009; Lee et al. 2008; Moore et al. 2005). To determine whether MCAK association with non-kinetochore MTs was negatively regulated by the chromosome-derived signal, cells were depleted of Nuf2, which prevented the formation of kinetochore-MT attachments ((DeLuca et al. 2002) and data not shown). Interestingly, in Nuf2-depleted cells, MCAK staining on MT plus-ends was still decreased in the vicinity of chromosomes (Supplemental Fig. S1c; Fig. 1e), demonstrating that MCAK associated with plusends of non-kinetochore MTs is spatially regulated by the chromosome-derived signal. This conclusion was further validated by triple staining of unperturbed prometaphase cells for MCAK, EB1, and the kinetochore marker BubR1 (Supplemental Fig. S2a).

To confirm that MCAK is negatively regulated by a chromosome-derived signal, in contrast to a signal coming from the centrosomes or the spindle, dynein heavy chain (DHC) was depleted, which results in strong separation of centrosomes from the DNA in late G2 and early mitosis (Bolhy et al. 2011; Splinter et al. 2010). Indeed, in DHCdepleted cells, MCAK could clearly be observed on MTs plus-ends in the vicinity of centrosomes but was largely absent from plus-ends near the chromosomes (Fig.2a). MCAK localization was also analyzed during prophase, at which time the nuclear envelope is still intact and therefore the chromosomes cannot signal to MT plus-ends yet. Before nuclear envelope breakdown (NEB), MCAK localized to all MT plus-ends, independently of their position within the cell (Supplemental Fig. S2b; Fig. 2b, c), but as soon as NEB was observed (as determined by chromosomes that had moved out of the nucleus), MCAK association with MT tips in the vicinity of chromosomes was strongly reduced (Supplemental Fig. S2c; Fig. 2b, c). These experiments not only show that the signal which negatively regulates MCAK binding to plus-ends is coming from chromosomes but also that the pool of MCAK binding to non-kinetochore MT plus-ends is affected, as at these very early time points after NEB very few kinetochore-MTs exist. Together, these results demonstrate that chromosomes generate a signal in mitosis that negatively regulates MCAK binding to non-kinetochore MT plus-ends.

MCAK mutants in which the Aurora B phosphorylation sites are mutated to phospho-mimicking residues, no longer bind to MT plus-ends (Honnappa et al. 2009; Moore et al. 
A MCAK
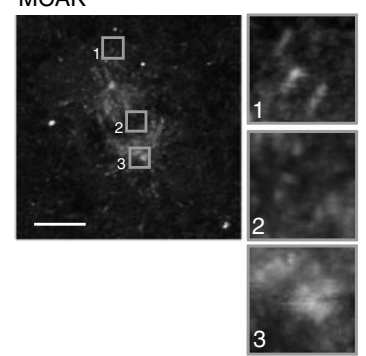

C
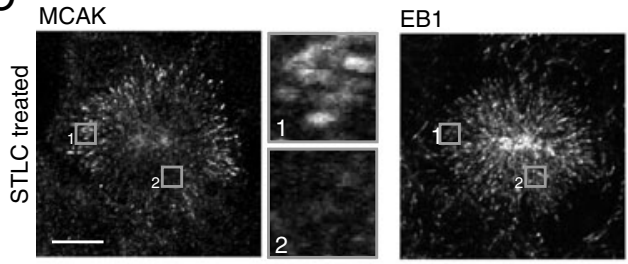

E
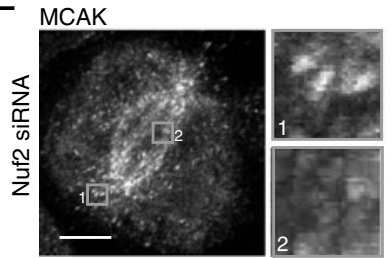
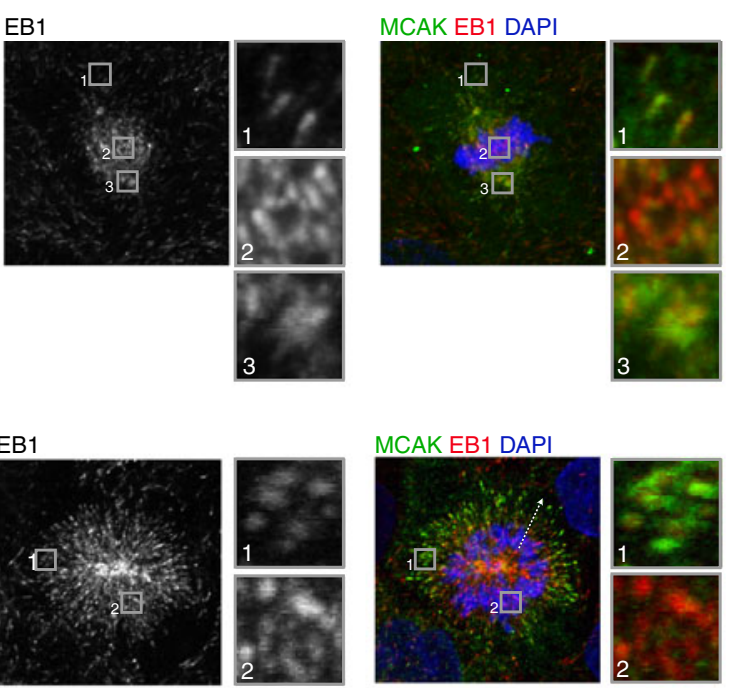

EB1

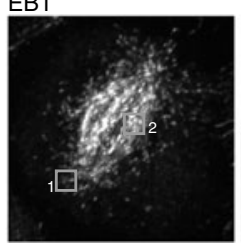

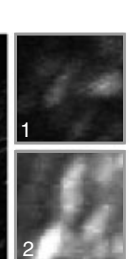
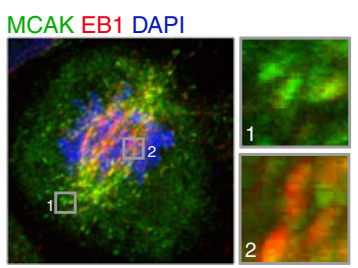

B

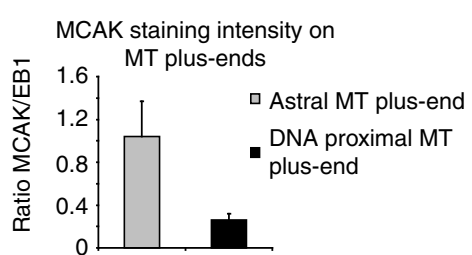

D

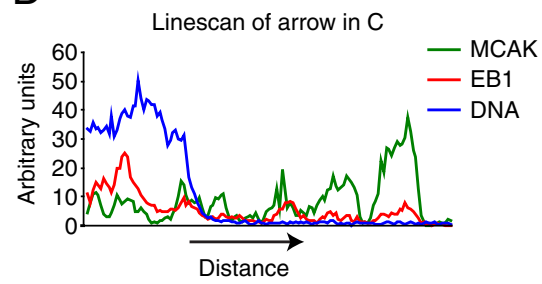

MCAK staining intensity on MT plus-ends

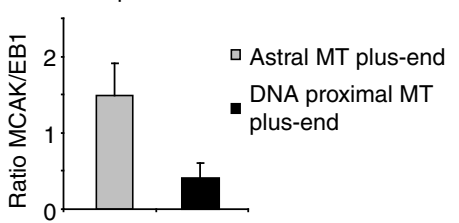

Fig. 1 MCAK binding to plus-ends of non-kinetochore MTs is reduced around chromosomes. a-c U2OS cells were fixed and stained with indicated antibodies. Boxed regions are shown enlarged on the right-hand side of the image (a, c). Cells in (a) were untreated and in (c) were treated with STLC for $1 \mathrm{~h}$ prior to fixation. b Line scans were generated through the MT plus-end and quantification was performed as described in the "Materials and methods." $\mathbf{d}$ A line scan was made

2005), suggesting that Aurora B might negatively regulate MCAK association with MT plus-ends. Furthermore, Aurora B is specifically activated at chromosomes (Kelly et al. 2007) and thus positioned such that it could generate the chromosome-derived signal that negatively regulates MCAK binding to plus-ends. To test whether the chromosome-derived signal that negatively regulates MCAK is dependent on Aurora B, the localization of MCAK was analyzed in cells treated with the Aurora B inhibitors AZD1152 (Mortlock et al. 2007) or ZM447439 (Ditchfield et al. 2003) together with the proteasome inhibitor MG132 to prevent mitotic exit. Strikingly, while MCAK localization was absent from MT plus-ends around chromosomes in cells treated with MG132 alone, MCAK localized prominently to all MT plus-ends after inhibition of Aurora B (Supplemental Fig. S3a-c; Fig. 3a). Importantly, depletion of the small GTPase Ran did not affect the spatial distribution of MCAK (Supplemental Fig. S3d; Fig. 3a), while it did block binding of the known Ran target HURP to chromosome-proximal MTs, which in turn was not affected by inhibition of Aurora B (Supplemental Fig. along the arrow in (c) and the MCAK, EB1, and DAPI signals are plotted. e Cells were transfected with Nuf2 siRNA and were fixed and stained with indicated antibodies after $48 \mathrm{~h}$. Quantification was performed as in (b). Graphs in (b) and (e) represent an average of four (b) and three (e) independent experiments with five cells analyzed per experiment and ten plus-ends quantified per cell. Error bars represent standard deviation. All scale bars indicate $5 \mu \mathrm{m}$

S3e-g). Similarly, in dynein-depleted cells, Aurora B inhibition restored MCAK association with MT plus-ends near chromsoomes (Fig. 3b). Detailed quantitative analysis of MCAK localization at multiple sites in the cell, with increasing distance from chromosomes, revealed that the Aurora B-dependent signal decreased in strength with increasing distance from chromosomes (Fig. 3c), suggesting that a gradient of Aurora B activity surrounds chromosomes. Together, these results show that Aurora B kinase activity generates a signal around chromosomes that negatively regulates MCAK binding to plus-ends. Furthermore, these results show that Aurora B and Ran act independently of each other, consistent with previous studies in Xenopus egg extracts (Kelly et al. 2007; Maresca et al. 2009; Sampath et al. 2004).

As Aurora B inhibits binding of MCAK to MT plus-ends around chromosomes in (pro)metaphase and MCAK binding to plus-ends enhances its MT-destabilizing activity (Montenegro Gouveia et al. 2010), inhibition of Aurora B is expected to destabilize MT plus-ends near chromosomes. Indeed, we found a $>20 \%$ decrease $(P<0.05)$ in plus-ends 
A MCAK

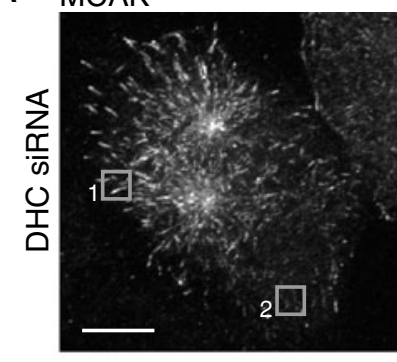

B
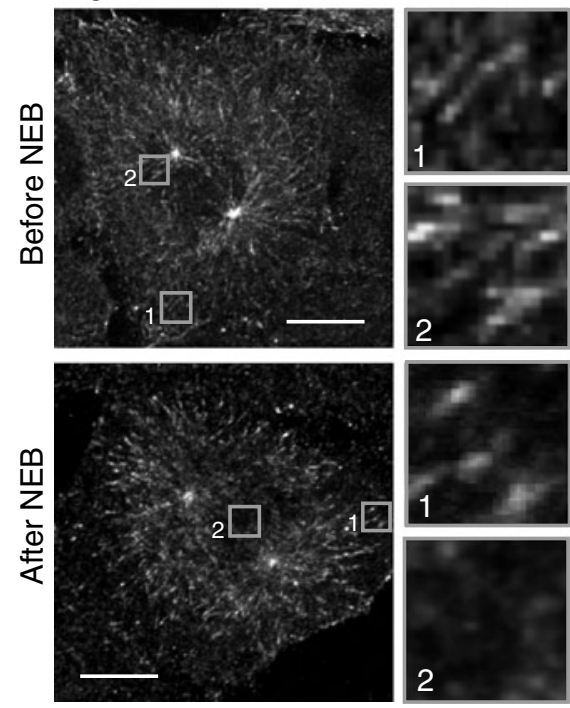

C

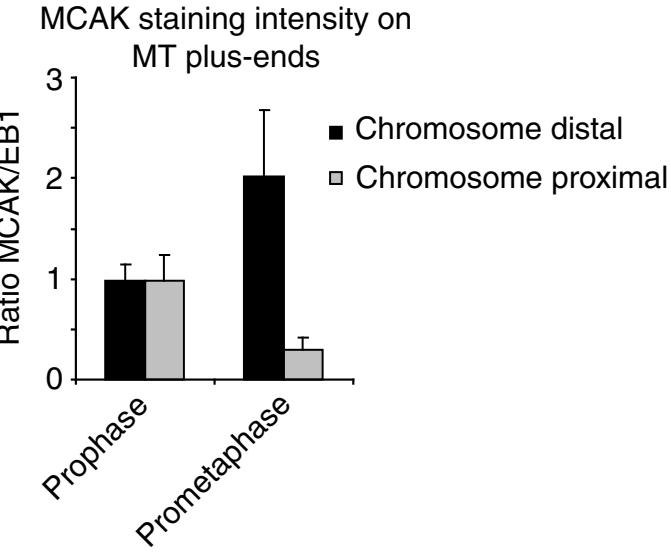

Fig. 2 A chromosome-derived signal regulates MCAK association with non-kinetochore MTs after NEB. a U2OS cells were transfected with siRNA against DHC and were re-transfected after $24 \mathrm{~h}$. Seventytwo hours after the first transfection, cells were fixed and stained with indicated antibodies. b-c Cells were fixed and stained with indicated antibodies. Prophase and early prometaphase cells were identified based on the DAPI staining. All boxed regions are shown enlarged on

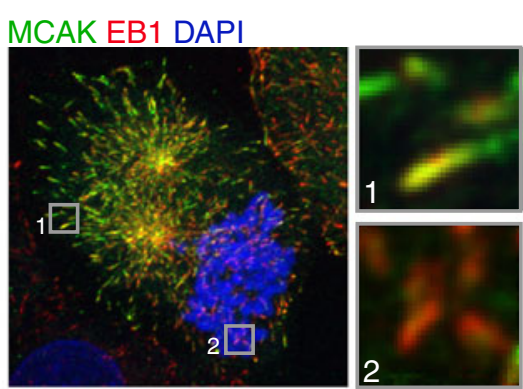

\section{EB1}
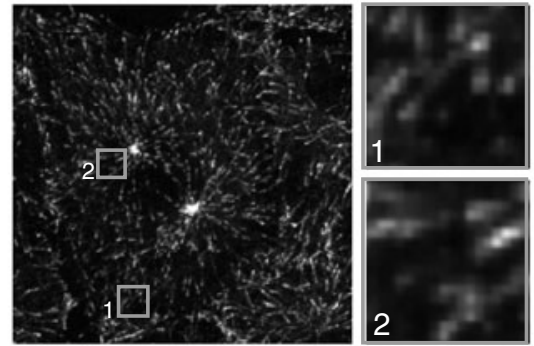

MCAK EB1 DAPI
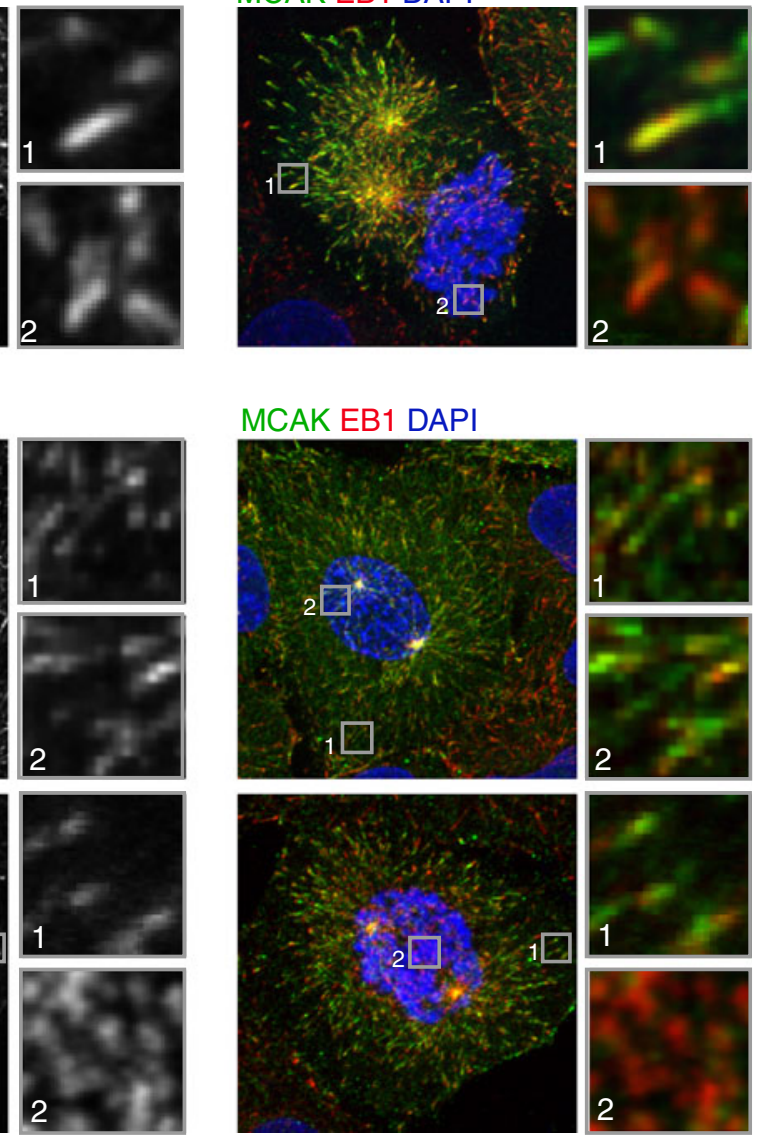

2

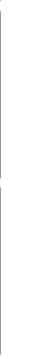

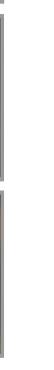


anaphase MCAK localized robustly to astral MTs plusends, as well as MT plus-end at the spindle pole-proximal side of the chromosomes, but was largely displaced from MT plus-end in the spindle midzone (Supplemental Fig. S4). These results suggest that regulation of MCAK binding to MT plus-ends by Aurora B continues in anaphase, and that negative regulation of MCAK by Aurora $\mathrm{B}$ in anaphase could promote local MT stabilization, providing a novel mechanism by which Aurora B could promote anaphase MT midzone stability.

\section{Discussion}

Here, we used MT plus-end localization of MCAK to examine the spatial distribution of Aurora $\mathrm{B}$ activity during spindle assembly. We find that a potent Aurora B-dependent signal, which negatively regulates MCAK association with MT plus-ends, surrounds the chromosomes (Fig. 4b, c). As MCAK binding to MT plus-ends is controlled through direct phosphorylation by Aurora $\mathrm{B}$ and inhibition of Aurora B eliminates the spatial distribution of MCAK, these results indicate that the observed spatially restricted localization of MCAK is the consequence of localized Aurora B kinase activity. Taken together, these results provide the first visualization of restricted Aurora B around chromosomes and indicate that Aurora B spatially regulates the dynamics of non-kinetochore MTs during spindle assembly.

Efficient spindle assembly requires both MT formation around chromosomes/kinetochores and capture of centrosomally nucleated MTs at kinetochores (Walczak and Heald 2008). Both types of MTs could benefit from local inhibition of MCAK-dependent MT depolymerization around chromosomes. First, MT seeds nucleated around chromosomes need to undergo substantial growth before they can contribute to the kinetochore fiber, which would be promoted by local inhibition of the MT depolymerase MCAK. Indeed, MT formation near chromosomes was shown to be blocked by MCAK in the absence of Aurora B activity (Sampath et al. 2004; Tulu et al. 2006). Second, MTs growing from the centrosomes can undergo random fluctuations of growth and shrinkage until they encounter a kinetochore. Selective stabilization of MTs near chromosomes (which has been experimentally observed (Athale et al. 2008; Dogterom et al. 1996)), will result in a bias of MTs growing towards chromosomes and could increase the MT capture rate at kinetochores. Aurora B likely acts in concert with Ran to generate a robust spatial cue at chromosomes, which facilitates spindle assembly.

How could chromosome-associated Aurora B generate a spatial cue around chromosomes? First, it is possible that after activation at chromosomes (Kelly et al. 2007), Aurora
$\mathrm{B}$ is released into the cytoplasm, where it phosphorylates its substrates while diffusing away from chromosomes and being rapidly inactivated, resulting in a gradient of Aurora B activity around chromosomes. Alternatively, active Aurora B could remain associated with chromosomes and phosphorylate cytoplasmic MCAK, which, in turn, diffuses away and is rapidly dephosphorylated, resulting in a similar gradient of Aurora B substrate phosphorylation. Finally, it is possible that Aurora B only phosphorylates and displaces MCAK from MT plus-ends physically contacting the chromosomes (as Aurora B may be most active when bound to both chromosomes and MTs (Tseng et al. 2010)). We did, however, find MCAK negative MT plus-ends that were clearly not contacting chromosomes, arguing against this model.

MCAK localizes to many different sites in dividing cells, including spindle poles, centromeres, kinetochores, chromosome arms and plus-ends of growing MTs (Moore et al. 2005; Walczak et al. 1996; Wordeman and Mitchison 1995) and its activity and/or localization to these sites is regulated by several kinases (Andrews et al. 2004; Knowlton et al. 2006; Lan et al. 2004; Ohi et al. 2004; Sanhaji et al. 2010; Zhang et al. 2011; Zhang et al. 2008; Zhang et al. 2007). Much attention has focused on regulation of chromosomeassociated MCAK and its role in regulating kinetochore MTs (Andrews et al. 2004; Knowlton et al. 2006; Lan et al. 2004; Ohi et al. 2004; Zhang et al. 2007). Here, we show that Aurora B has an additional role in regulating MCAK on non-kinetochore MTs. As several other kinases, like Aurora A, Plk1 and CDK1 were also shown to phosphorylate MCAK (reviewed in Tanenbaum et al. 2011), it will be important to determine how these phosphorylation events are regulated in space and time and which pool of MCAK is controlled by which kinase. Furthermore, examination of individual phosphorylation sites, as well as combinations of sites will be essential since even phosphorylation by a single kinase on multiple sites can have different effects on MCAK function (Zhang et al. 2007).

In conclusion, these results reveal that chromosomeassociated Aurora B provides a spatial cue around chromosomes which specifically displaces MCAK from MT plusends, resulting in a local increase in MT stability. This Aurora B-dependent spatial cue likely acts in concert with the Ran signaling gradient to contribute to efficient spindle assembly and formation of kinetochore-MT attachments.

\section{Materials and methods}

Cell culture, transfection, and drug treatments

Cell culture and transfection were performed as described previously (Tanenbaum et al. 2006). MCAK, Ran, and 


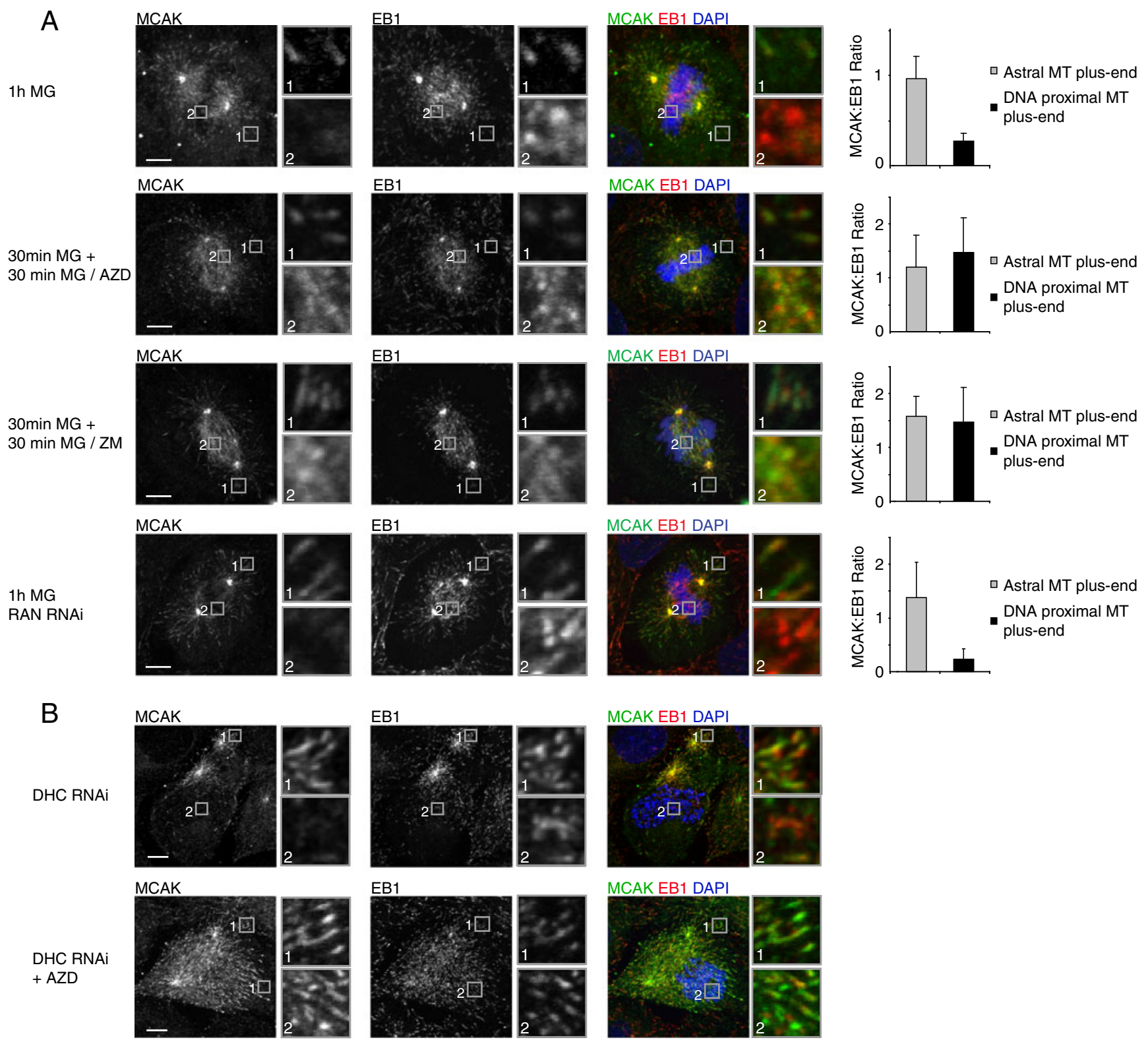

C
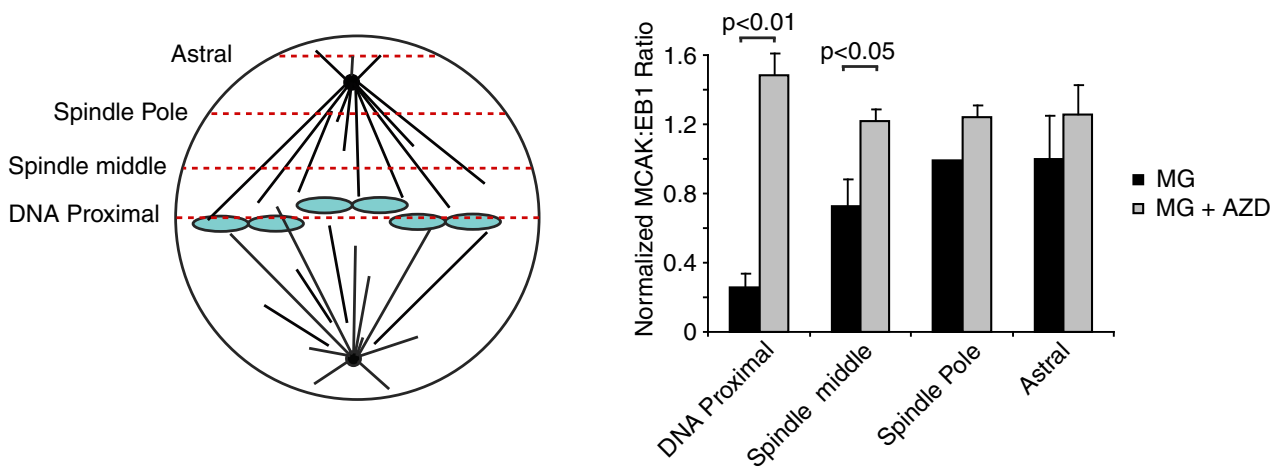

Nuf2 siRNA's were OTP SMARTpools from Dharmacon; DHC siRNA was described previously (Draviam et al. 2006; Tanenbaum et al. 2008). MG132 (Sigma) was used at
$5 \mu \mathrm{M}, \mathrm{ZM} 447439$ (AstraZeneca) was used at $2 \mu \mathrm{M}$ and AZD-1152 (Selleck) was used at $100 \mathrm{nM}$. S-Trityl-L-cystein (Sigma) was used at $5 \mu \mathrm{M}$. 
4Fig. 3 Localized Aurora B activity spatially regulates MCAK association with MT plus-ends. a-b U2OS cells were transfected with siRNA's where indicated and $48 \mathrm{~h}$ after transfection, cells were treated either with $1 \mathrm{~h} \mathrm{MG132}$ or first with MG132 for $30 \mathrm{~min}$ to allow for chromosome alignment and then with AZD1152 (100 nM) or ZM447439 $(2 \mu \mathrm{M})$ together with MG132 for $30 \mathrm{~min}$. Cells were then were fixed and stained with indicated antibodies. Line scans were generated through the MT plus-end and quantification was performed as described in the "Materials and methods." Graphs show averages of three independent experiments with five cells and ten plus-ends scored per cell. b U2OS cells were transfected with DHC siRNA and cells were re-transfected after $24 \mathrm{~h}$. Seventy-two hours after the first transfection cells were either treated with AZD1152 for $3 \mathrm{~h}$ or were left untreated. Cells were then fixed and stained with indicated antibodies. In (c), the normalized ratio of MCAK/EB1 (see "Materials and methods") was determined at four positions in the cell, as indicated in the left panel. The graph is the average of four independent experiments. All error bars represent standard deviations between experiments. Scale bars indicate $5 \mu \mathrm{m}$

\section{Immunofluorescence}

Cells were grown on 10-mm glass coverslips and fixed with cold methanol for $5 \mathrm{~min}$, unless stated otherwise. Antibodies were used in the following dilutions: MCAK (Walczak et al. 1996), 1:1,500; EB1 (BD), 1:500; HURP (Sillje et al. 2006), 1:1,000; and BubR1 (sheep, a kind gift of G. Kops), 1:1,000. Primary antibodies were incubated overnight at room temperature and secondary antibodies
(Alexa 488 and 561, Molecular Probes) were incubated for $1 \mathrm{~h}$ at room temperature. DAPI was added before mounting using Vectashield (Vectorlabs). Images were acquired on a Zeiss LSM510 META confocal microscope (Carl Zeiss) with a Plan Apochromat $\times 63$ NA 1.4 objective. $Z$-planes were acquired with $1-\mu \mathrm{m}$ intervals. Brightness and contrast were adjusted with Photoshop 6.0 (Adobe). Images are maximum intensity projections of all $Z$-planes.

\section{Quantification of MCAK staining}

Images of EB1 and MCAK staining were acquired using identical microscope settings for all samples. To determine the background signal, three line scans were performed close to, but not overlapping with, a microtubule plus-end using the Zeiss LSM510 software and the average intensities were calculated. To determine the amount of EB1 and MCAK at a microtubule plus-end, EB1 comets were randomly selected and line scans were made with a fixed length through the comet. Average intensities of both EB1 and MCAK were then calculated. In each experiment, five cells were scored, in which ten-microtubule plus-ends were randomly selected. For Fig. 3c, the ratio of MCAK/ EB1 was determined at four regions in the cell and the ratio at the spindle middle in control cells was set to 1 . Ratios at other regions were then normalized to the spindle middle
A

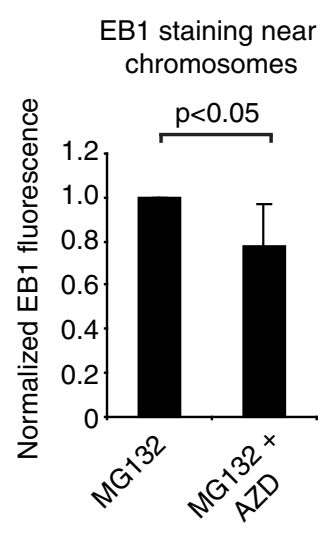

B

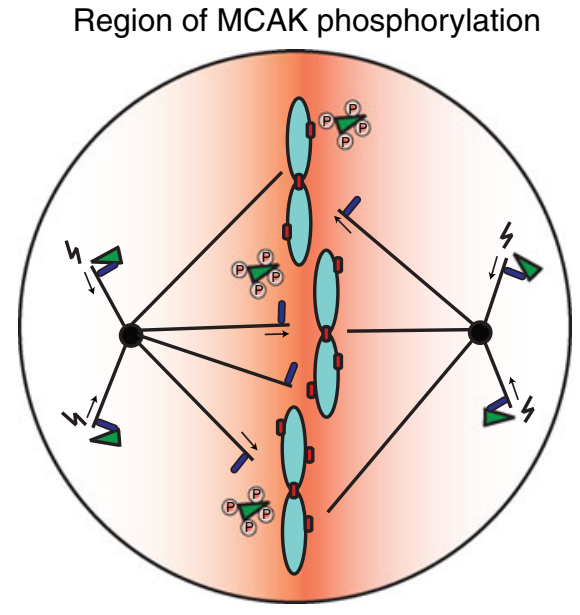

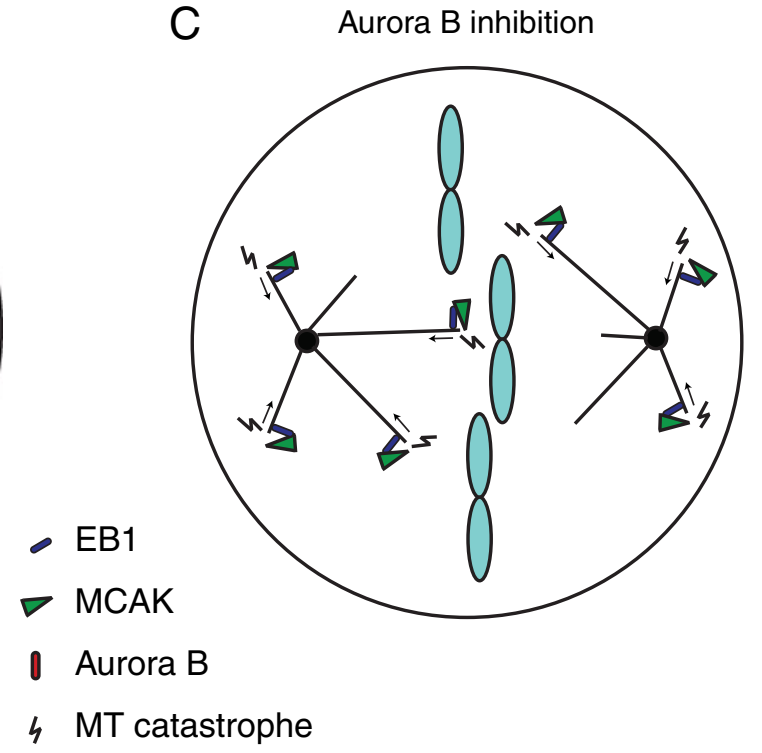

Fig. 4 Aurora B stabilizes MT plus-ends near chromosomes. a Cells were treated with either $1 \mathrm{~h} \mathrm{MG132}$ or first 30 min with MG132 to allow for chromosome alignment to the metaphase plate and then 30 min with MG132+100 nM AZD1152. Cells were then fixed and stained for EB1. Total EB1 staining intensity was determined in the area of the chromosomes and values were corrected for background staining. For each independent experiment the staining intensity of the control was set to 1 . Graph represents average of four independent experiments with ten cells analyzed per experiment, and error bars represent standard deviation. b-c Model. b MCAK binds to MT plusends through its association with EB1 and induces MT catastrophes. However, in the vicinity of the chromosomes MCAK becomes phosphorylated by Aurora B and can no longer bind EB1, locally decreasing MT catastrophes. The orange gradient represents the region in which MCAK is present in its phosphorylated form and thus, cannot bind to MT plus-ends. c Aurora B inhibition eliminates the reduced binding of MCAK to DNA-proximal MT plus-ends, resulting in destabilization of chromosome-proximal MT plus-ends 
value of controls. Averages and standard deviations between experiments were then calculated based on these normalized values.

Acknowledgments We would like to thank Claire Walczak for providing the MCAK antibody, Geert Kops for the BubR1 antibody, Erich Nigg for the HURP antibody, and the members of the Medema group for helpful discussions. This work was supported by the Division for Earth and Life Sciences (ALW) and the Netherlands Genomic Initiative, both with financial aid of the Netherlands Organization for Scientific Research (NWO).

Open Access This article is distributed under the terms of the Creative Commons Attribution Noncommercial License which permits any noncommercial use, distribution, and reproduction in any medium, provided the original author(s) and source are credited.

\section{References}

Andrews PD, Ovechkina Y, Morrice N, Wagenbach M, Duncan K, Wordeman L, Swedlow JR (2004) Aurora B regulates MCAK at the mitotic centromere. Dev Cell 6:253-268

Athale CA, Dinarina A, Mora-Coral M, Pugieux C, Nedelec F, Karsenti E (2008) Regulation of microtubule dynamics by reaction cascades around chromosomes. Science 322:12431247

Bolhy S, Bouhlel I, Dultz E, Nayak T, Zuccolo M, Gatti X, Vallee R, Ellenberg J, Doye V (2011) A Nup133-dependent NPC-anchored network tethers centrosomes to the nuclear envelope in prophase. J Cell Biol 192:855-871

Clarke PR, Zhang C (2008) Spatial and temporal coordination of mitosis by Ran GTPase. Nat Rev Mol Cell Biol 9:464-477

DeLuca JG, Moree B, Hickey JM, Kilmartin JV, Salmon ED (2002) hNuf2 inhibition blocks stable kinetochore-microtubule attachment and induces mitotic cell death in HeLa cells. J Cell Biol 159: 549-555

Ditchfield C, Johnson VL, Tighe A, Ellston R, Haworth C, Johnson T, Mortlock A, Keen N, Taylor SS (2003) Aurora B couples chromosome alignment with anaphase by targeting BubR1, Mad2, and Cenp-E to kinetochores. J Cell Biol 161:267-280

Dogterom M, Felix MA, Guet CC, Leibler S (1996) Influence of M-phase chromatin on the anisotropy of microtubule asters. J Cell Biol 133:125-140

Draviam VM, Shapiro I, Aldridge B, Sorger PK (2006) Misorientation and reduced stretching of aligned sister kinetochores promote chromosome missegregation in EB1- or APC-depleted cells. EMBO J 25:2814-2827

Fuller BG, Lampson MA, Foley EA, Rosasco-Nitcher S, Le KV, Tobelmann P, Brautigan DL, Stukenberg PT, Kapoor TM (2008) Midzone activation of aurora $\mathrm{B}$ in anaphase produces an intracellular phosphorylation gradient. Nature 453:1132-1136

Gadea BB, Ruderman JV (2006) Aurora B is required for mitotic chromatin-induced phosphorylation of Op18/Stathmin. Proc Natl Acad Sci U S A 103:4493-4498

Honnappa S, Gouveia SM, Weisbrich A, Damberger FF, Bhavesh NS, Jawhari H, Grigoriev I, van Rijssel FJ, Buey RM, Lawera A et al (2009) An EB1-binding motif acts as a microtubule tip localization signal. Cell 138:366-376

Jiang K, Wang J, Liu J, Ward T, Wordeman L, Davidson A, Wang F, Yao X (2009) TIP150 interacts with and targets MCAK at the microtubule plus ends. EMBO reports 10:857-865
Kalab P, Weis K, Heald R (2002) Visualization of a Ran-GTP gradient in interphase and mitotic Xenopus egg extracts. Science 295:2452-2456

Kalab P, Pralle A, Isacoff EY, Heald R, Weis K (2006) Analysis of a RanGTP-regulated gradient in mitotic somatic cells. Nature 440:697-701

Kelly AE, Sampath SC, Maniar TA, Woo EM, Chait BT, Funabiki H (2007) Chromosomal enrichment and activation of the aurora B pathway are coupled to spatially regulate spindle assembly. Dev Cell 12:31-43

Kirschner M, Mitchison T (1986) Beyond self-assembly: from microtubules to morphogenesis. Cell 45:329-342

Knowlton AL, Lan W, Stukenberg PT (2006) Aurora B is enriched at merotelic attachment sites, where it regulates MCAK. Curr Biol 16:1705-1710

Koffa MD, Casanova CM, Santarella R, Kocher T, Wilm M, Mattaj IW (2006) HURP is part of a Ran-dependent complex involved in spindle formation. Curr Biol 16:743-754

Lan W, Zhang X, Kline-Smith SL, Rosasco SE, Barrett-Wilt GA, Shabanowitz J, Hunt DF, Walczak CE, Stukenberg PT (2004) Aurora B phosphorylates centromeric MCAK and regulates its localization and microtubule depolymerization activity. Curr Biol 14:273-286

Lee T, Langford KJ, Askham JM, Bruning-Richardson A, Morrison EE (2008) MCAK associates with EB1. Oncogene 27:2494 2500

Liu D, Vader G, Vromans MJ, Lampson MA, Lens SM (2009) Sensing chromosome bi-orientation by spatial separation of aurora B kinase from kinetochore substrates. Science 323:1350-1353

Maresca TJ, Groen AC, Gatlin JC, Ohi R, Mitchison TJ, Salmon ED (2009) Spindle assembly in the absence of a RanGTP gradient requires localized CPC activity. Curr Biol 19:12101215

Montenegro Gouveia S, Leslie K, Kapitein LC, Buey RM, Grigoriev I, Wagenbach M, Smal I, Meijering E, Hoogenraad CC, Wordeman $\mathrm{L}$ et al (2010) In vitro reconstitution of the functional interplay between MCAK and EB3 at microtubule plus ends. Curr Biol 20:1717-1722

Moore AT, Rankin KE, von Dassow G, Peris L, Wagenbach M, Ovechkina Y, Andrieux A, Job D, Wordeman L (2005) MCAK associates with the tips of polymerizing microtubules. J Cell Biol 169:391-397

Mortlock AA, Foote KM, Heron NM, Jung FH, Pasquet G, Lohmann JJ, Warin N, Renaud F, De Savi C, Roberts NJ et al (2007) Discovery, synthesis, and in vivo activity of a new class of pyrazoloquinazolines as selective inhibitors of aurora B kinase. J Med Chem 50:2213-2224

Niethammer P, Bastiaens P, Karsenti E (2004) Stathmin-tubulin interaction gradients in motile and mitotic cells. Science 303:1862-1866

Ohi R, Sapra T, Howard J, Mitchison TJ (2004) Differentiation of cytoplasmic and meiotic spindle assembly MCAK functions by Aurora B-dependent phosphorylation. Mol Biol Cell 15:28952906

Ruchaud S, Carmena M, Earnshaw WC (2007) Chromosomal passengers: conducting cell division. Nat Rev Mol Cell Biol 8:798-812

Sampath SC, Ohi R, Leismann O, Salic A, Pozniakovski A, Funabiki $\mathrm{H}$ (2004) The chromosomal passenger complex is required for chromatin-induced microtubule stabilization and spindle assembly. Cell 118:187-202

Sanhaji M, Friel CT, Kreis NN, Kramer A, Martin C, Howard J, Strebhardt K, Yuan J (2010) Functional and spatial regulation of mitotic centromere-associated kinesin by cyclin-dependent kinase 1. Mol Cell Biol 30:2594-2607 
Sillje HH, Nagel S, Korner R, Nigg EA (2006) HURP is a Ranimportin beta-regulated protein that stabilizes kinetochore microtubules in the vicinity of chromosomes. Curr Biol 16:731-742

Splinter D, Tanenbaum ME, Lindqvist A, Jaarsma D, Flotho A, Yu KL, Grigoriev I, Engelsma D, Haasdijk ED, Keijzer N et al (2010) Bicaudal D2, dynein, and kinesin-1 associate with nuclear pore complexes and regulate centrosome and nuclear positioning during mitotic entry. PLoS Biol 8:e1000350

Tanenbaum ME, Galjart N, van Vugt MA, Medema RH (2006) CLIP-170 facilitates the formation of kinetochore-microtubule attachments. EMBO J 25:45-57

Tanenbaum ME, Macurek L, Galjart N, Medema RH (2008) Dynein, Lis1 and CLIP-170 counteract Eg5-dependent centrosome separation during bipolar spindle assembly. EMBO J 27:3235-3245

Tanenbaum ME, Medema RH, Akhmanova A (2011) Regulation of localization and activity of the microtubule depolymerase MCAK. BioArchitecture 1:80-87

Tseng BS, Tan L, Kapoor TM, Funabiki H (2010) Dual detection of chromosomes and microtubules by the chromosomal passenger complex drives spindle assembly. Dev Cell 18:903-912

Tulu US, Fagerstrom C, Ferenz NP, Wadsworth P (2006) Molecular requirements for kinetochore-associated microtubule formation in mammalian cells. Curr Biol 16:536-541
Vader G, Medema RH, Lens SM (2006) The chromosomal passenger complex: guiding Aurora-B through mitosis. J Cell Biol 173:833-837

Walczak CE, Heald R (2008) Mechanisms of mitotic spindle assembly and function. Int Rev Cytol 265:111-158

Walczak CE, Mitchison TJ, Desai A (1996) XKCM1: a Xenopus kinesin-related protein that regulates microtubule dynamics during mitotic spindle assembly. Cell 84:37-47

Wordeman L, Mitchison TJ (1995) Identification and partial characterization of mitotic centromere-associated kinesin, a kinesinrelated protein that associates with centromeres during mitosis. $\mathrm{J}$ Cell Biol 128:95-104

Zhang X, Lan W, Ems-McClung SC, Stukenberg PT, Walczak CE (2007) Aurora B phosphorylates multiple sites on mitotic centromere-associated kinesin to spatially and temporally regulate its function. Mol Biol Cell 18:3264-3276

Zhang X, Ems-McClung SC, Walczak CE (2008) Aurora A phosphorylates MCAK to control ran-dependent spindle bipolarity. Mol Biol Cell 19:2752-2765

Zhang L, Shao H, Huang Y, Yan F, Chu Y, Hou H, Zhu M, Fu C, Aikhionbare F, Fang G et al (2011) PLK1 phosphorylates mitotic centromere-associated kinesin and promotes its depolymerase activity. J Biol Chem 286:3033-3046 